\title{
The Scattering of Electromagnetic Waves by Plasma Oscillations
}

\author{
By Noboru HOKKYO \\ Department of Physics, Osaka City University
}

(Read. May. 15, 1956 ; Received June 23, 1956)

\begin{abstract}
Theory of plasma oscillation is applied to the problem of the scattering of $\sim 1000 \mathrm{Mc}$ waves in the $E$ layer of the ionosphere. It is assumed that a region of the ionosphere having an irregular or turbulent character contains "oscillating domains" or "coherent units" in which free electrons perform synchronous oscillations. These domains are shown to be effective in scattering waves of frequencies $\sim(c / v) \omega_{p}$, where $\omega_{p}$ is the plasma frequency, $v$ the mean thermal speed of electrons, and $c$ the velocity of light. A rough estimate indicates that the scattered wave may be observable for a proper setup in the experiment, and that its angular dependence is $\left(\sin 3 \frac{1}{2} 0\right)^{-1}, 0$ being the scattering angle.
\end{abstract}

\section{Introduction}

Several theoretical studies [1] [2] concerning the problem of the galactic radiofrequency radiation, as well as of the disturbed solar radiation point towards an explanation in terms of plasma oscillations excited by turbulent streams of ionized gases. These theories are based on the fact that the plasma oscillation, the organized oscillation of free electrons as a whole, interacts with the radiation. It is therefore of interest to investigate the possibility that electromagnetic waves are scattered by the plasma oscillations.

An electromagnetic wave is not scattered in passing through a medium in which atoms (electrons) are fairly uniformly distributed, even though the medium contains a great many atoms which individually produce scattered wavelets. On the other hand, fluctuations of density in the medium cause the scattering of light, on account of imperfect destructive interferences between the scattered wavelets. An incident wave of wave length $L$ can only be scattered if, in the Fourier expansion of density fluctuations, waves with spatial periods of the order of $L$ occur. An attempt to explain the scattering of meter waves in the $E$ layer of the ionosphere has been made by Villars and Weisskopf [3]. They proposed an idea that there are external causes which constantly produce large eddies of a certain dimension $L_{0}$ and that these are divided soon into smaller and smaller eddies. The dividing process ends with that eddy size $L_{s}$ at which the effect of molecular viscosity is large enough to dissipate the energy of the eddy into heat. Consequently, their theory is applicable for incident waves of wave lengths intermediate between $L_{0}$ and $L_{s}$, that is, meter waves. 
In the present paper we would like to point out that the scattering of higher frequency waves $\left(\sim 10^{3} \mathrm{Mc}\right)$ is also made possible by electrons under the action of periodic electric potentials associated with the plasma oscillations. The ionospheric turbulence as suggested by Villars and Weisskopf will be certain to cause the plasma of the ionosphere to start oscillation [4]. When electric fields are introduced by incomplete space charge neutralization due to disturbances, the highly mobile free electrons automatically respond to the forces in such a way as to shield out the fields, and a systematic oscillation of charged region is set up. The frequency of the organized oscillation of the electron gas as a whole is known to be [3] $\omega_{\text {org }}=\left[\omega_{p}{ }^{2}+(3 \kappa T / m)\right]^{1 / 2}$ $\cdot(2 \pi / \lambda), \omega_{p}$ being the plasma frequency defined by $\omega^{2} p=4 \pi e^{2} \times$ (density of electrons) $/ \mathrm{m}$ and $\lambda$ referring to the wave length. The initial deviation in velocity and density distribution of electrons from equilibrium due to the disturbance will build up longitudinal waves of a given frequency not always restricted to the frequency given by the above dispersion relation. In time, however, these will tend to get out of phase with each other, and only the organized plasma waves for which the frequency is given by the dispersion relation continue to contribute to macroscopic averages such as the mean electric potential. It should be noted that in the $E$ layer of the ionosphere $\omega_{\text {org }} \simeq \omega_{p}$ (for large $\lambda$ ) $\simeq 10^{7} \mathrm{sec}^{-1}$ is two powers of ten above the mean frequency of collision which an electron makes with molecules, so that the plasma oscillates many times before the organized motion is lost by close collision of electrons with molecules (collisions between the electrons are neglected for the electronic densities encountered in the ionosphere). The plasma oscillations will be excited most effectively in the turbulent region. Electrons which enter the region in which the plasma oscillations are highly excited differ from those outside the region in that they suffer an oscillatory change in their velocities. We shall see that these electrons scatter light at the expence of their extra momenta arising from their oscillatory motions and that the scattered wavelets from a set of electrons confined to a common trough of the plasma potential (coherent unit) interfere constructively.

\section{Scattering by Plasma Oscillation}

In a plasma an assembly of electrons undergoes an organized oscillation, the plasma oscillation, brought about by the Coulomb forces between charges. The plasma oscillation or the plasma wave, can be analyzed into a surperposition of plane waves which can have all wave lengths, down to limiting waves whose wave lengths are of the same order of magnitude of the Debye length [4] [6].

Each individual electron is in a state of continual fluctuation in its motion under the influence of a periodic potential associated with the plasma wave, and the electron can be deprived of its extra momentum and energy by an electromagnetic wave which traverses the medium. That is, a part of the monentum and energy of the plasma wave can be transferred into incoming electromagnetic wave through the intermediary of electrons. We might describe this by saying that the light is scattered by a plasma oscillation. As the plasma is a system of a set of individual electrons 
interacting with the collective plasma oscillations with the uniform background of positive charge, let us imagine as the smallest copy of a plasma an 'atom' consisting of an electron plus a plasma wave. The plasma is now looked upon as containing a great many such atoms which individually can produce scattered wavelets. The energy of the atom is degenerate in the sense that a given value of the total energy is distributed in different ways on the electron and the plasma wave, although these states will be different in momentum from each other corresponding to different distributions of the total energy. The scattering process consists in the absorption of a primary radiation and the emission of a secondary radiation. The scattering atom may be left either in its initial state, or in some other state. In particular, if the atom returns to a state which is equal to the initial state in energy but different in momentum, the emerging radiation carries away a definite amount of momentum (but not energy) from the atom, so that the process will appear as a reflection from the atom.

\section{Determination of the Optimum Frequency}

Since we are interested in the scattering of $\mathrm{cm}$ waves whose wave lengths are much shorter than the linear dimension of the turbulence such as the eddy, we can get a better idea of how the plasma oscillation works in the scattering by taking an idealized plasma wave of infinitely long wave length. The plasma wave now varies only in time and therefore exchanges energy, but not momentum with electrons. An electron acted on by a periodic perturbation of the plasma potential exchanges, say absorbs, quantum $\hbar \omega_{p}$ and makes a transition to a virtual state, in which energy and momentum are not related by a free particle equation $E=p^{2} / 2 m$, and backs again to the initial state, reemitting $\hbar \omega_{p}$, in a time of the order of $\tau=1 / \omega_{p}$. If in the time $\tau$ available from the uncertainty principle a radiation falls upon the electron in the virtual state and gives a kick to it so that the electron receives such an amount of momentum $\hbar \Delta \mathbf{k}$ as to satisfy $E+\hbar \omega_{p}=(\mathbf{p}+\hbar \Delta \mathbf{k}) / 2 m$, the free particle equation, the electron becomes a real one and stays in the new energy state indefinitely while one quantum is lost from the plasma oscillation, the 'atom' making an internal conversion. The radiation then gets momentum $-\hbar \Delta \mathbf{k}$, and is deflected with an angle of deflection, say $\theta . \quad \theta$ is determined from the above free particle relation, or simply from

to be

$$
\Delta \mathbf{k v} \simeq \pm \omega_{p}
$$

$$
\Delta k=2 k \sin \theta=\omega_{p} / v \cos (\Delta \hat{\mathbf{k v}})
$$

where $\mathbf{v}=\mathbf{p} / m, \pm$ corresponds to the absorption ( + ) and the emission ( - ) of the longitudinal quantum, and $k$ refers to the wave number of the incident wave.

If space occupied by electrons were unlimited, the momentum change $\Delta \mathbf{k}$ of electrons would be completely determined by the requirement that it be equal to the momentum change of the incident light. However, since we are considering those electrons that are contained in a certain region of space of, say, linear size $l$ (which will be equated to the wave length of the plasma wave), $\Delta \mathbf{k}$ is not defined to within 
$(2 \pi / l)$. Now it may be seen that the solutions $\Delta \mathbf{k}_{s}$ of (1) center densely around the value $\Delta k=\omega_{p} / v$ with $\Delta \mathbf{k v}=0$. Therefore setting $\cos (\Delta \mathbf{k v})=1$ in (2) gives the angular frequency at which the deflection occurs as

$$
\omega=\frac{\omega_{p}}{2 \sin \frac{\theta}{2}} \frac{c}{v}
$$

For the $E$ layer of the ionosphere where $\omega_{p} \simeq 10^{7} \mathrm{sec}^{-1}$ corresponding to the mean electron density and $v \simeq 10^{7} \mathrm{~cm} \mathrm{sec}^{-1}$, the mean thermal speed, we have

$$
\omega / 2 \pi \simeq 10^{3} \mathrm{Mc} / \mathrm{sec},
$$

which is higher than the penetration frequency, $\omega_{p} / 2 \pi$, by a factor $(c / v)$.

Lastly we notice from (1) with fixed $\Delta \mathbf{k}$ that if, for instance, electrons of velocity $\mathbf{v}$ scatter light by damping the plasma oscillation, those of velocity $-\mathbf{v}$ do the same by exciting the oscillation.

\section{Interaction Hamiltonian}

The scattering of a light quantum is the second order action of the vector potential $\mathbf{A}$ of the electromagnetic field. Since $\omega_{p} \ll \omega$, we start with the interaction Hamiltonian [5]

$$
H=\sum_{i} \frac{e^{2}}{2 m c^{2}} \mathbf{A}^{2}\left(\mathbf{x}_{i}\right)
$$

which describes the scattering of a light quantum on a set of free electrons in the nonrelativistic limiting case. So long as we do not know from which electron the light quantum is scattered, we sum over the electron coordinates. The Fourier component of $\mathbf{A}\left(\mathbf{x}_{i}\right)$ responsible for the scattering $\mathbf{k}_{0} \rightarrow \mathbf{k}$ is

$$
A e^{i(\mathbf{k}-\mathbf{k} 0) \mathbf{x} i}=\frac{4 \pi \hbar c^{2}}{\omega}\left(\boldsymbol{e}_{0} \boldsymbol{e}\right) e^{i\left(\mathbf{k}_{0}-\mathbf{k}\right) \mathbf{x} i}
$$

where $\boldsymbol{e}_{0}, \boldsymbol{e}$ represent unit vectors in the direction of polarization of two light quanta of frequency $\omega$ and of wave vectors $\mathbf{k}_{0}, \mathbf{k}$.

Our system is a set of individual electrons interacting with the collective plasma oscillations. Since a photon, striking an electron and being scattered, causes the internal conversion of the atom, that is, a non-reversing absorption or a real emission by the electron of the quantum $\hbar \omega_{p}$ which must be made up by a change of the energy or the momentum of the electron, we consider the expression

$$
\frac{e^{2}}{2 m c^{2}} \sum_{i}^{v n_{i}} A e^{i(\mathbf{k} 0-\mathbf{k}) \mathbf{x} i}\left[\sum_{s} e^{-i \Delta \mathbf{k} i s \mathbf{x} i}\right]
$$

where $\left|\mathbf{k}-\mathbf{k}_{0}\right|=\omega_{p} /\left\langle v>_{A} V, V n_{i}\right.$ is the number of atoms contained in a volume $V=l^{3}$, that is, the number of individual electron degrees of freedom $(\div 3)$ and $\sum_{s}$ denotes the summation over all the solutions $\Delta \mathbf{k}_{i s}$ of (1) with $\mathbf{v}=\mathbf{v}_{i}$. In case of large $V n_{i}$ (7) reduces to

$$
H_{c}=\frac{e^{2}}{2 m c^{2}} \sum_{i}^{V n_{s e}} A e^{i\left(\mathbf{k}_{0}-\mathbf{k}-\Delta \mathbf{k} i\right) \mathbf{x} i},
$$


the summation over $i$ being this time only for those $V n_{s c}$ electrons for which

$$
\left|\mathbf{k}_{0}-\mathbf{k}-\Delta \mathbf{k}_{i}\right| \lesssim(2 \pi / l)
$$

where

$$
\Delta k_{i} \simeq \omega_{p} / v_{i}
$$

When $l \rightarrow \infty$ (9) simply expresses the law of conservation of momentum. In obtaining (8) we have neglected a number of terms which are multiplied by a phase factor with a nonvanishing argument $(>(2 \pi / l) x), \exp \left[i\left(\mathbf{k}_{0}-\mathbf{k}-\Delta \mathbf{k}\right) \mathbf{x}_{i}\right]$. There is a strong tendency for various such terms entering into (7) to cancel out, because atoms (electrons) are distributed over a wide variety of positions in the region of linear size $l$ and there would be just as many atoms in the region where $\exp \left[i\left(\mathbf{k}_{0}-\mathbf{k}-\Delta \mathbf{k}\right) \mathbf{x}_{i}\right]$ is positive as where it is negative [6]. These argument, however, will not apply when the exponential function does not vary appreciably inside the region; hence, the condition (9). In this way $H_{c}$ becomes practically independent of the electron coordinates, and despite the random location of the electrons the scattered wavelets from various electrons all add up cumulatively (interfere constructivelyf to produce a scattered wave.

One can obtain a simple physical picture of this. An incident wave $\psi$ which falls upon each atom varies with the position of the atom as $\exp \left(i \mathbf{k}_{0} \mathbf{x}_{i}\right)$. The scattering of the light by our atom is like the first order action of a perturbing potential $U$ varying in space as $\exp \left(-i \Delta \mathbf{k} \mathbf{x}_{i}\right)$ :

$$
U\left(\mathbf{x}_{i}\right)=\left(\boldsymbol{e}^{2} / m\right)\left(2 \pi \hbar / \omega_{p}\right)\left(\boldsymbol{e}_{0} \boldsymbol{e}\right) \exp \left(-i \Delta \mathbf{k} \mathbf{x}_{i}\right)
$$

The scattered wave is the sum of a set of wavelets originating at the positions of the atoms. Since each atom contributes according to the product of the potential $U\left(\mathbf{x}_{i}\right)$, and the incident wave $\psi\left(\mathbf{x}_{i}\right)$, an outgoing wavelet that spreads out from $\mathbf{x}_{i}$ as $\exp \left[i \mathbf{k}\left(\mathbf{x}-\mathbf{x}_{i}\right)\right]$ is weighted with the amplitude factor $U\left(\mathbf{x}_{i}\right) \psi\left(\mathbf{x}_{i}\right)$. Thus the scattered wave at a point $\mathbf{x}$ is obtained by adding up the contributions of each atom with a phase of $\exp (i \mathbf{k x}) \exp \left[i\left(\mathbf{k}_{0}-\mathbf{k}-\Delta \mathbf{k}\right) \mathbf{x}_{i}\right]$.

Hence, if $\mathbf{k}_{0}-\mathbf{k}-\Delta \mathbf{k} \simeq 0$ is fulfilled various wavelets from various atoms add in phase with each other:

$$
\sum_{i} A \exp (i \mathbf{k x}) \exp \left[i\left(\mathbf{k}_{0}-\mathbf{k}-\Delta \mathbf{k}\right) \mathbf{x}_{i}\right]=V \boldsymbol{n}_{s c} A \exp (i \mathbf{k x})
$$

We observe that the square of $H$ and hence the scattering cross section is proportional to $n^{2}$. Hence our process will bring about a noticeable effect in case of large $\boldsymbol{n}_{\boldsymbol{s}}$. It should be noted, however, that, since the collective components of the density fluctuations of electrons have been expressed in terms of plasma waves, the number per unit volume of individual electron degrees of freedom is reduced from $3 n$ to $3 n_{i}=3 n-n_{D}$. Here $n$ is the density of electrons and $n_{D}$ the number of collective degrees of freedom given by [6]

$$
n_{D}=(4 \pi / 3)\left(1 / \lambda_{D}\right)^{3}
$$

where $\lambda_{D}$ denotes 'Debye length.' Since $\lambda_{D} \simeq 1 \mathrm{~cm}$ in our case, we are justified in 
setting $n_{i} \simeq \boldsymbol{n} \simeq 10^{5} \mathrm{~cm}^{-3}$. We also note that not all individual electrons are effective in deflecting incident waves in a given direction, instead a fraction of them, $n_{s c}=n_{i} \Delta \Omega$ $/ 4 \pi$, where $\Delta \Omega$ is a small solid angle centering about $\Delta \mathbf{k} \simeq\left(\mathbf{k}_{0}-\mathbf{k}\right)$ is effective. It may be seen that $\Delta \Omega / 4 \pi \simeq 1 / \Delta k l$, * where $l=V^{1 / 3}$ is the linear dimension of the effective volume $V$ in which coherent scattering can take place.

\section{Efficiency for the Scattering}

From $H_{c}$ we obtain in the usual way, the scattering cross section per unit volume for the scattering of light by a set of 'atoms'

$$
\sigma / V \simeq r_{0}^{2} n_{s c}^{2} V=r_{0}^{2}(n / \Delta k)^{2} l
$$

where a factor of order unity is omitted. $r_{0}$ is the classical electron radius defined by $r_{0}=e^{2} / m c^{2}$. Since the plasma oscillations cannot be sustained for wave lengths shorter than a critical distance known as the Debye length, $\lambda_{D} \simeq<v_{A_{A} v} / \omega_{p}$ (which is of the order of the distance traveled during the period of an oscillation by an electron moving with the mean thermal speed), the actual wave lengths will be much larger than $\lambda_{D}$. We infer roughly: $l=L_{s} \simeq 10^{2} \lambda_{D}$, that is, the dimension of the smallest eddy. Inserting this into (14) and setting $\Delta k=\omega_{p} / v \simeq 1 \mathrm{~cm}^{-1}$ corresponding to the scattering of $1000 \mathrm{Mc}$ wave give

$$
\sigma / V \simeq 10^{-12} \mathrm{~cm}^{-1}
$$

The useful scattering volume depends upon the geometry for the scattering which corresponds to the set up in the experiment. By using the same geometry as Bailey et al. (for the scattering of meter waves) the ratio $P_{r} / P_{t}$ of the power received to the power transmitted becomes

$$
\frac{P_{r}}{P_{t}}=\frac{4 \sigma}{V} \frac{b A}{\sin (\theta / 2) D^{2}}=4 r_{0}^{2} l\left(\frac{n}{\Delta k}\right)^{2} \frac{b A}{D^{2} \sin (\theta / 2)},
$$

where $b$ is the thickness of the scattering layer having an irregular or turbulent character, $A$ the aperture of the receiving antenna and $D$ the ray length which is practically identical with the surface transmission distance. The following set of values of the parameters are taken from the paper by Villars and Weisskopf [3]:

$$
\begin{gathered}
A=3 \times 10^{6} \mathrm{~cm}^{2}, \quad D=10^{8} \mathrm{~cm}, \quad b=5 \times 10^{5} \mathrm{~cm}, \\
\theta=\text { scattering angle }=24^{\circ} .
\end{gathered}
$$

Substituting these values, we get

$$
\frac{P_{r}}{P_{t}} \simeq 10^{-18}
$$

It is of interest to compare (17) with the experimental data for the scattering of $50 \mathrm{Mc}$ wave which indicate a ratio [3] [7]: $0.36 \times 10^{-18}$.

We actually should expect, however, a somewhat smaller result than (17), because the scattering volume will not be filled everywhere with the oscillating domains.

* From (1) $(\Delta k+2 \pi / l) v \cos \alpha=\omega_{p}=\Delta k v\left(\alpha=\Delta \Omega^{1 / 2}\right)$ and $\cos \alpha=\Delta k /(\Delta k+2 \pi / l) \simeq 1-2 \pi / \Delta k l$. Since $\cos \alpha \simeq 1-\alpha^{2} / 2$, we get $\Delta \Omega=4 \pi / \Delta k l$. 


\section{Summary}

Our argument was based on the fact that the energy of the 'atom' is degenerate with regard to changes of the momentum when the total energy of the system of electron plus plasma wave is kept fixed. Under the strong periodic perturbation, that is, when the amplitude of the plasma wave becomes large in the atom, reversing changes in the distribution of the energy (exchanges of $\hbar \omega_{p}$ between the electron and the plasma wave) occur, so that the electron in the atom must be thought of as covering several virtual states centering about a real state at once. We have thus far assumed that when a light quantum falles upon the atom a non-reversing transition between degenerate levels, which we called the internal conversion, occurs; the momentum thereby liberated is just as large as to deflect the $1000 \mathrm{Mc}$ waves. A further possibility to be taken into consideration is that the atom may be left after the scattzring in its initial state, when no permanent change in the distribution of energy between the electron and the plasma wave (net exchange of $\hbar \omega_{p}$ ) does occur. In other words, the electron in the atom may alone scatter light, the plasma oscillation playing no role. Then we must put $\mathbf{k}_{0}-\mathbf{k} \simeq \Delta \mathbf{k}=0$ in (8) in case of uniform distribution of electrons, which means that the light after the scattering travels off in the same direction as the incident one. The only effect of the interaction Hamiltonian $\boldsymbol{H}$ is therefore that of refraction, a change in the phase velocity as it passes through the medium. On the other hand, when there are such fluctuations of the electron concentration that there are more electrons in the region where $\exp \left[i\left(\mathbf{k}_{0}-\mathbf{k}\right) \mathbf{x}_{i}\right]$ is positive (negative) than where it is negative (positive), $H$ produces a scattered wave due to imperfect destructive interferences between the scattered wavelets. Thus the presence of density fluctuations whose spatial periods are of the order $L$ is essential for the scattering of waves of wave lengths $\sim L<c / \omega_{p}$. This is the content of the scattering of meter waves.

One now obtains a general feature of the scattering in the $E$ layer of the ionosphere of waves above the penetration frequency as follows. An electromagnetic wave which enters a region of the ionosphere, in which the electron concentration fluctuates, experiences a changing index of refraction and is scattered. When the wave length of the incident wave becomes comparable or smaller than $L_{s} \simeq 10^{2} \mathrm{~cm}$, the linear dimension of the smallest eddy, the scattering will fall off rapidly. As the wave length decreases further from this value, however, and the frequency approaches three powers of ten above the penetration frequency, the wave will again begin to be scattered by the plasma oscillations. Thus, tied up with the theory of the scattering of meter waves, our argument leads to the selective reflection of electromagnetic waves impinging on the ionosphere.

\section{Acknowledgment}

The author is greatly indebted to Professor S. Hayakawa for many enlightening discussions and suggestions during the progress of this work. 


\section{References}

[1] A. Unsöld, Phys. Rev. 82, 857 (1951)

[2] S. Hayakawa and N. Hokkyo, Prog. Theor. Phys. 15, 193 (1956)

[3] F. Villars and V.F. Weisskopf, Phys. Rev. 94232 (1954)

[4] D. Bohm and E.P. Gross, Phys. Rev. 75, 1851, 1864 (1949)

[5] Heitler, Quantum Theory of Radiation

[6] D. Bohm and D. Pines, Phys. Rev. 92.609 (1953)

[7] D.K. Bailey et al., Phys. Rev. 86. 141 (1952) 